\title{
Questionário de Auto-avaliação Vocal: Instrumento Epidemiológico de Controle da Síndrome Disfônica Ocupacional em Professores
}

\section{Vocal Self Assessment Questionnaire: Epidemological Control Tool of the Ocupational Dysphonic Syndrome in Professors}

\author{
Sandra Irene Cubas de Almeida*, Paulo Pontes**, Marco Antonio Bussacos***, Luciano Neves****, \\ Fabiana Zambon $* * * * *$.
}

\footnotetext{
* Doutora em Medicina. Chefe do Setor de Relações do Trabalho MTE-Gerência Norte/SP.

*** Professor Titular do Departamento de Otorrinolaringologia e Distúrbios da Comunicação Humana UNIFESP.

**** Pesquisador da Fundacentro.

***** Mestre em Otorrinolaringologia.Douturando em Otorrinolaringologia UNIFESP.

****** Fonoaudiologista. Fonoaudióloga do SINPRO/SP.

Instituição: Ministério do Trabalho e Emprego - Gerência Norte. São Paulo / SP - Brasil

Endereço para correspondêcia: Sandra Irene Cubas de Almeida - Av. General Ataliba Leonel, 2764 - Parada Inglesa-São Paulo / SP - Brasil - CEP: 02242-000 - Telefone: (+55 11) 2973-8927 - E-mail: sandra.almeida@mte.gov.br

Artigo recebido em 8 de Abril de 2010. Artigo aprovado em 29 de Maio de 2010
}

\section{RESUMO}

Introdução:

Objetivo:

Método:

Resultados:

Conclusão:

Palavras-chave:

\section{SUMMARY}

Introduction: Objective:

Method:

Results:

Conclusion:

Keywords:
A síndrome disfônica é uma doença ocupacional de alta prevalência na população de professores. A fim de abordar preventivamente a agressão vocal, procurou-se padronizar um questionário de autoavaliação para professores de todos os níveis de ensino, incluindo além dos sintomas clínicos também os fatores da organização do trabalho.

Foi realizado um estudo de corte transversal em 328 professores de 4 instituições de ensino da região norte de São Paulo, aos quais aplicou-se um questionário de auto-avaliação elaborado pela Comissão Tripartite de Normatização para a Voz Profissional.

Determinou-se a prevalência dos sintomas clínicos que compõem a síndrome disfônica, assim como retratou-se a organização do trabalho e qualidade de vida desta população.

O questionário aplicado mostrou-se satisfatório na descrição e elaboração de parâmetros com o objetivo de implementação de programas preventivos para populações expostas à sobrecarga profissional do uso da voz. Estudo de caso.

disfonia, medicina do trabalho, riscos ocupacionais, saúde do trabalhador, pesquisa.

The dysphonic syndrome is an occupational disease of high prevalence in the professor population. In order to broach preventively the vocal aggression, it was tried to standardize a self assessment questionnaire to professors of all educational level, including beyond the clinical symptoms also the factors of the work organization.

It was performed a study of transversal cut in 328 professors of 4 educational institutions of the northern of São Paulo, which it was applied a self assessment questionnaire elaborated by TRipartite Commission of Standardization for Professional Voice.

It was determined the prevalence of clinical symptoms that compound the dysphonic syndrome, as well as it was delineate the work organization and the quality of life of this population.

The questionnaire applied was showed itself satisfactory en the description and elaboration of parameters with the aim of to implement preventive programs to populations exposed to professional overload of the voice use. Case study.

dysphonia, occupational medicine, occupational risks, worker health, research. 


\section{INTRODUÇÃO}

A auto-avaliação do risco vocal é um procedimento inicial não invasivo, integrante do arsenal de metodologia diagnóstica, desenvolvida para ser aplicada ao controle epidemiológico e/ou individual, com o objetivo de detecção precoce e prevenção da síndrome disfônica ocupacional.

Síndrome disfônica ocupacional (SDO) refere-se ao conjunto de sinais e sintomas disfônicos desencadeados ou causados pelo uso da voz em atividades profissionais e que podem estar relacionados a outras causas como predisposição individual e fatores ambientais. (1)

A fase pré-patogênica pode culminar na alteração permanente da emissão vocal com o estabelecimento de laringopatias e, nesses casos, denominadas laringopatias relacionadas ao trabalho.

Os sinais e sintomas da síndrome disfônica são: dor ou irritação na garganta, sensação de corpo estranho, dor cervical, necessidade de pigarrear e a rouquidão sendo que medidas médicas preventivas poderão apresentar-se eficazes quando se consegue rastrear precocemente o conjunto citado. Portanto, a síndrome disfônica é um sinal de alerta na qual medidas e procedimentos específicos de ampla variabilidade, de acordo com as necessidades individuais, deverão ser desencadeados visando a prevenção da laringopatia.

A pesquisa clínica dos sinais/sintomas constitui, por consenso internacional, recomendado pela European Laryngological Society, uma das ações obrigatórias básicas mínimas da pesquisa em qualquer ação diagnóstica e/ou terapêutica sobre o sistema fonador. (2)

Os princípios básicos que devem ser seguidos na elaboração de uma auto-avaliação vocal são os seguintes:

1. Considerar que a função vocal é multidimensional.

2. Obter registros que possibilitem conclusões para a meta-análise.

3. Não excluir outros procedimentos que se fizerem necessários mas, precedê-los.

Vários trabalhos foram publicados no sentido de obter-se a prevalência da disfonia em professores utilizando-se questionários de auto-avaliação associados ou não a exames clínicos. O valor desta prevalência variou de 0,8 a $34 \%(3,4)$.

A partir de 1998 observou-se aumento de prevalência atingindo $70 \%$.

Em 1998, um grupo australiano avaliou professores e definiu através da elaboração de questionários a prevalência de queixas clínicas vocais nesta população (4).

O objetivo deste estudo é validar um questionário de auto-avaliação vocal dirigido à categoria profissional dos professores de todos os níveis de ensino para rastreamento dos sintomas da síndrome disfônica ocupacional e os fatores da organização de trabalho que interferem na história natural das laringopatias ocupacionais.

\section{MÉTOdO}

Foi realizado um estudo epidemiológico de corte transversal em 328 professores de 4 instituição de ensino da região norte de São Paulo de 2001 a 2002.

A estes professores foi aplicada a auto-avaliação elaborada pela Comissão Tripartite de Normatização da Voz Profissional (5).

Os quesitos para elaboração da auto-avaliação foram os seguintes:

1. Definir a identificação do indivíduo quanto ao sexo, idade, entidade mantenedora.

2. Organização do trabalho em relação ao vínculo empregatício com outras instituições, níveis de ensino para os quais leciona, carga de trabalho, pausas, número de alunos por sala, exercício de outras atividades profissionais.

3. Sintomas clínicos da Síndrome Disfônica.

4. Hábitos e qualidade de vida.

A aplicação era realizada nos períodos de aula nos intervalos e início das mesmas.

Precedendo a aplicação, realizou-se a verificação in loco das condições e fatores ambientais, assim como a organização do trabalho que foi detalhada no questionário de auto-avaliação.

Como parte do método realizamos a divulgação das técnicas de higiene vocal após a aplicação da auto-avaliação.

Considerando o objetivo de validar a auto-avaliação para definir o perfil populacional através da indicação da prevalência de sintomas da Síndrome Disfônica assim como determinar as características da população que podem atuar como risco na evolução da doença, o questionário deveria preencher os seguintes critérios:

1. Facilidade na compreensão das perguntas e respostas.

2. Tempo despendido para responder todo o conjunto.

3. Adequação do formato para análise estatística posterior.

4. Avaliação do próprio indivíduo quanto ao ambiente de trabalho e de sua organização. 
5. Rastreamento dos sintomas da síndrome disfônica ocupacional como manifestação pré-patogênica ou patogênese precoce na instalação de uma laringopatia ocupacional orgânica ou funcional incapacitante.

6. Consenso social de aprovação dos integrantes da Comissão Tripartite.

Os questionários foram aplicados para todos os professores que se encontravam na sala de professores no período que precedia o início das aulas e após o consentimento expresso e esclarecido do participante.

Após aprovação pelo Comitê de Ética Médica da UNIFESP e assinatura do Termo Esclarecido de Consentimento, os professores e a instituição recebiam orientações sobre higiene vocal, prevenção e controle médico do risco das laringopatias relacionadas ao trabalho. Os questionários eram aplicados pela equipe médica do MTE (Ministério do Trabalho e Emprego), UNIFESP (Universidade Federal de São Paulo) acompanhados por representantes do Sindicato dos Professores de São Paulo(SINPRO$\mathrm{SP})$, pelos serviços de segurança e saúde da própria instituição e membros da Comissão Interna de Prevenção de Acidentes (CIPA).

A operacionalização do método ocorreu in loco através da aplicação da auto-avaliação, verificação médicolegal do cumprimento das normas regulamentadoras que estabelecem o controle médico, controle dos riscos ambientais e as condições ergonômicas e organizacionais do trabalho (6).

O banco de dados foi criado no Excell e para as análises utilizou-se o Software SAS /SAT versão $9.12 \mathrm{em}$ análise estatística pela FUNDACENTRO.

\section{RESULTADOS}

Foram aplicados 328 questionários de auto-avaliação aos professores de quatro instituições de ensino universitário localizadas na região norte de São Paulo.

A auto-avaliação foi elaborada e dividida em quatro partes:

1. Identificação.

2. Organização do trabalho.

3. Sintomas clínicos.

4. Hábitos e qualidade de vida.

\section{DISCUSSÃO}

Ao iniciarmos o estudo sobre a prevalência e evolução da Síndrome Disfônica Ocupacional em professores, necessitávamos desenvolver uma metodologia de abordagem que nos mostrasse as características desta população e fornecesse elementos concretos para que pudéssemos propor medidas de controle eficazes.

Portanto, sendo a Síndrome Disfônica Ocupacional um conjunto de sinais e sintomas precoces no estabelecimento de uma laringopatia ocupacional, a abordagem inicial multidimensional nos fornece critérios para a prevenção das consequências patogênicas que culminam com a exclusão do indivíduo de seu ambiente profissional.

Uma das primeiras constatações que tivemos foi a discordância entre as exigências legais e as ações executadas nas instituições, ou seja, uma dissociação entre o controle médico proposto e executado e o risco vocal ocupacional conforme preconiza a legislação através dos princípios da Norma Regulamentadora 7 (7).

A partir deste trabalho nas instituições, os programas de controle de saúde ocupacionais passaram a contemplar medidas de saúde voltadas para os riscos da Síndrome Disfônica.

A revisão da literatura $(3,4,7)$ tem mostrado que esta é uma preocupação universal qual seja a abordagem da Síndrome Disfônica considerando a manifestação do indivíduo quanto aos aspectos de sua emissão vocal.

Um dos mais conhecidos trabalhos a respeito é a avaliação do Vocal Handicap Index que tem sido estudado e validado em outros idiomas além do inglês.

Através dos gráficos podemos definir características da população propondo ações de saúde e para os indivíduos testados, podemos acrescentar ao controle clínico os dados obtidos e segui-los prospectivamente.

Os gráficos evidenciam que nesta população de professores universitários há predomínio de homens (Gráfico 1) em faixa etária de mais de 35 anos (Gráfico 2) com dedicação ao ensino superior (Gráfico 3). Em relação à organização do trabalho, predominam as aulas de mais de 100 minutos (Gráfico 4) e o número de alunos por sala é de mais de 51 para a maioria (Gráfico 5). O sintoma dor ou irritação na garganta ocorre em $70 \%$, sendo mais frequente à noite (Gráfico 6 e 7). Sensação de corpo estranho é referida por $41 \%$ (Gráfico 8). Necessidade de pigarrear assim como dor no pescoço ocorre em 54\% (Gráfico 9 e 10). A rouquidão foi referida por $31 \%$ (Gráfico 11). Tabagismo foi declarado por 16\% (Gráfico 11) e alguma ingestão de álcool e medicamentos de uso contínuo por 20\% (Gráfico 12 e 13). Atividade esportiva é praticada por $50 \%$ dos avaliados. 


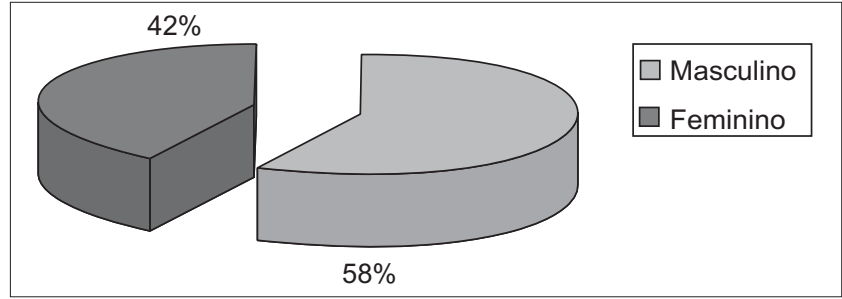

Gráfico 1. Prevalência de gênero.

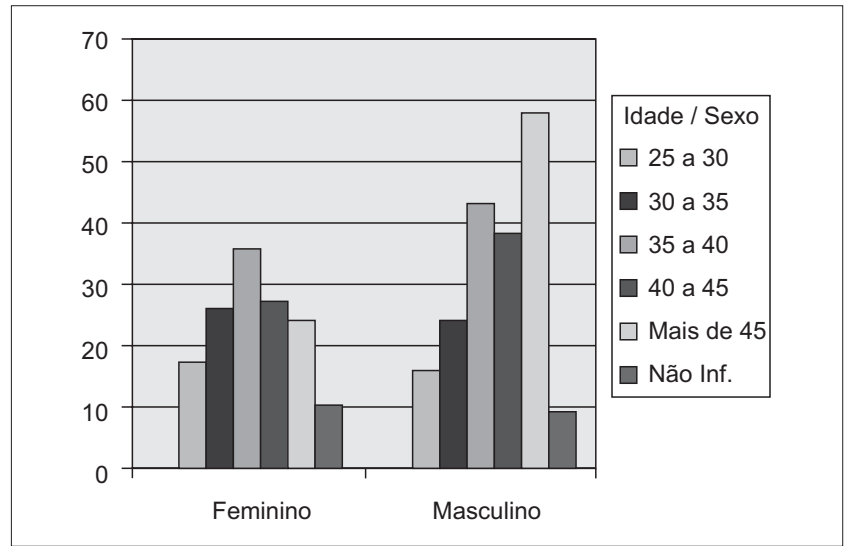

Gráfico 2. Distribuição de faixas etárias entre os gêneros.

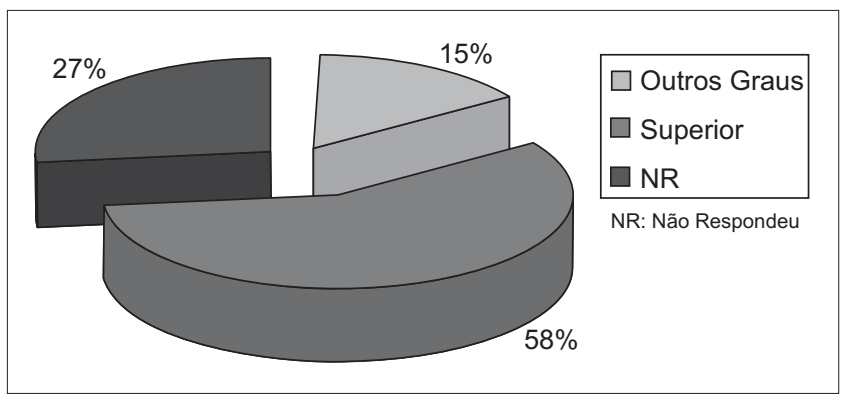

Gráfico 3. Prevalência de vínculos com outras instituições.

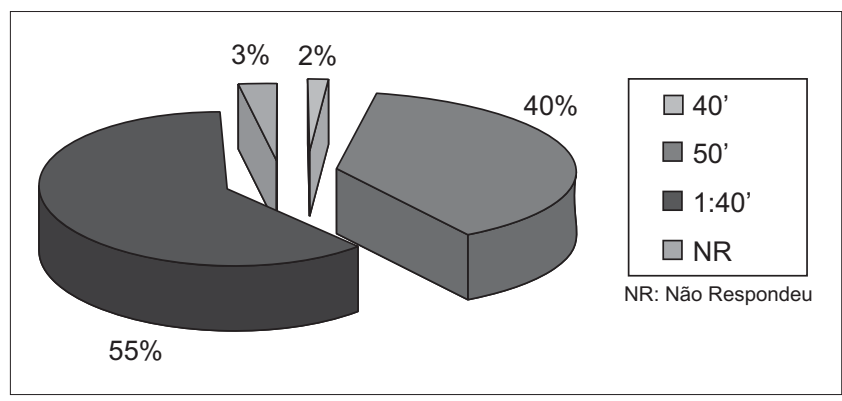

Gráfico 4. Prevalência da duração das aulas.

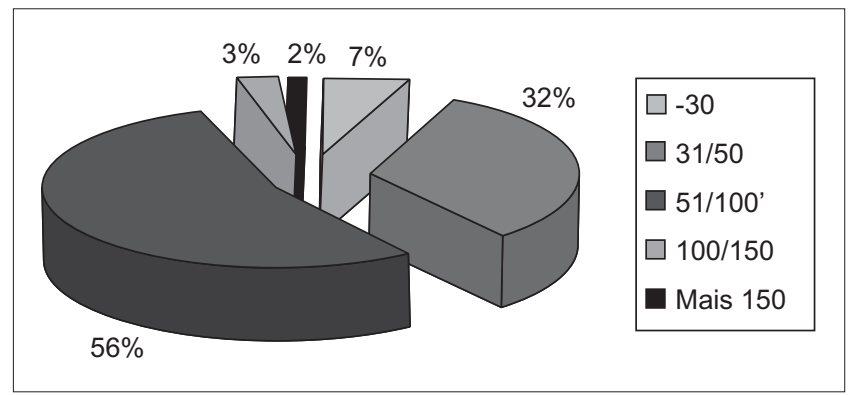

Gráfico 5. Prevalência de alunos por sala de aula.

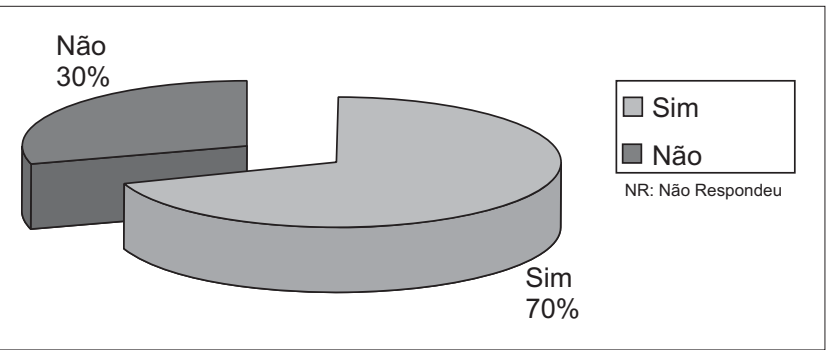

Gráfico 6. Prevalência do sintoma clínico dor ou irritação na garganta.

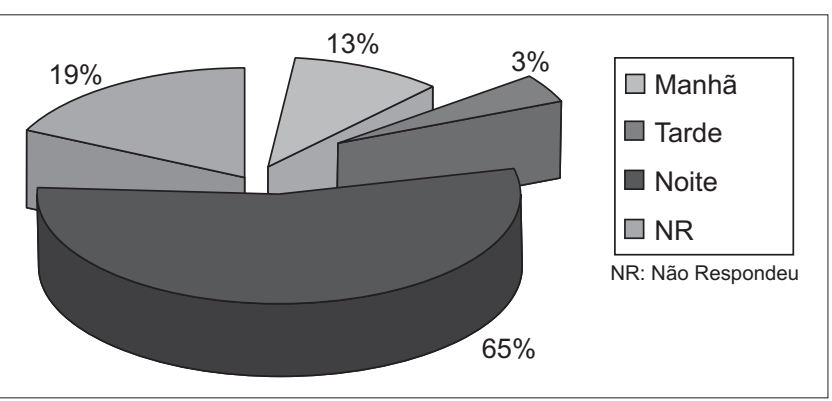

Gráfico 7. Prevalência do período do dia no qual há o sintoma clínico dor ou irritação na garganta.

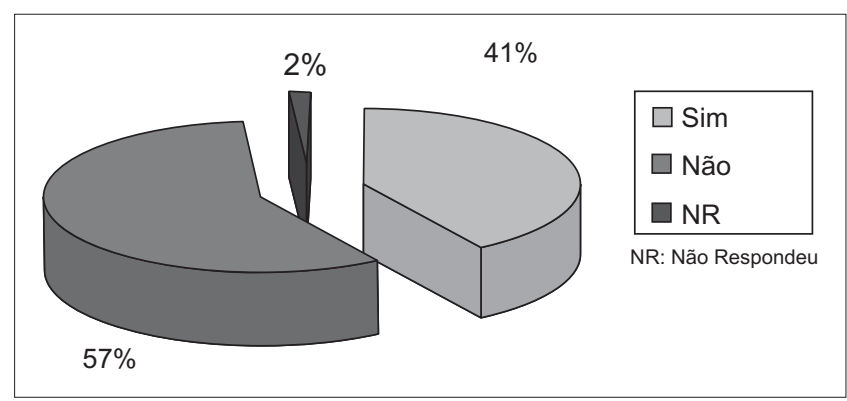

Gráfico 8. Prevalência do sintoma sensação de corpo estranho na garganta.

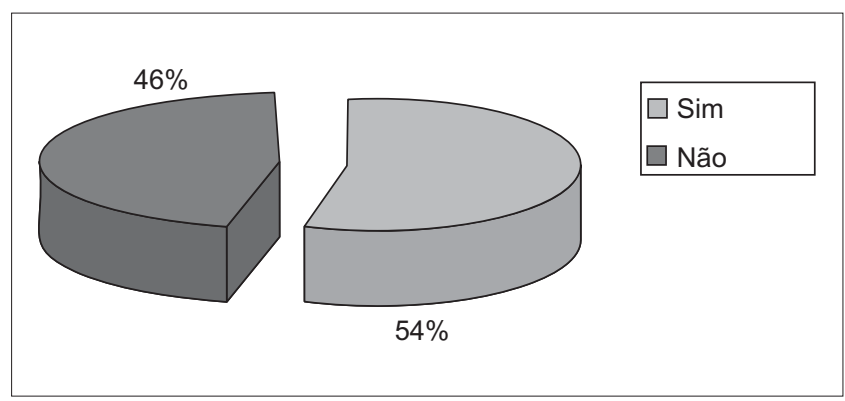

Gráfico 9. Prevalência do sintoma pigarro.

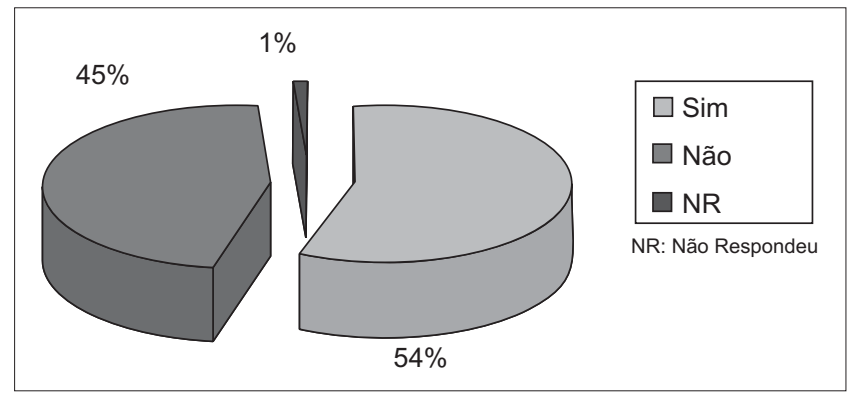

Gráfico 10. Prevalência do sintoma cervicalgia. 


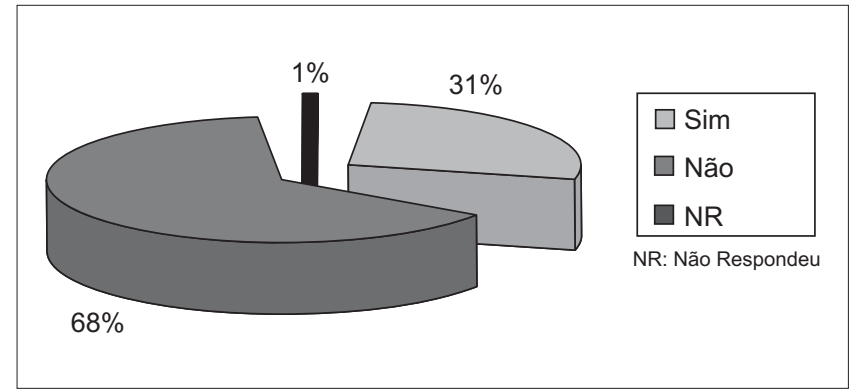

Gráfico 11. Prevalência de rouquidão.

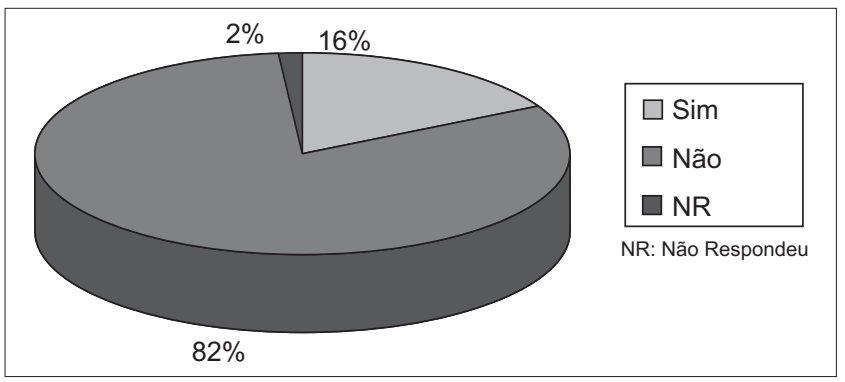

Gráfico 12. Prevalência de tabagismo.

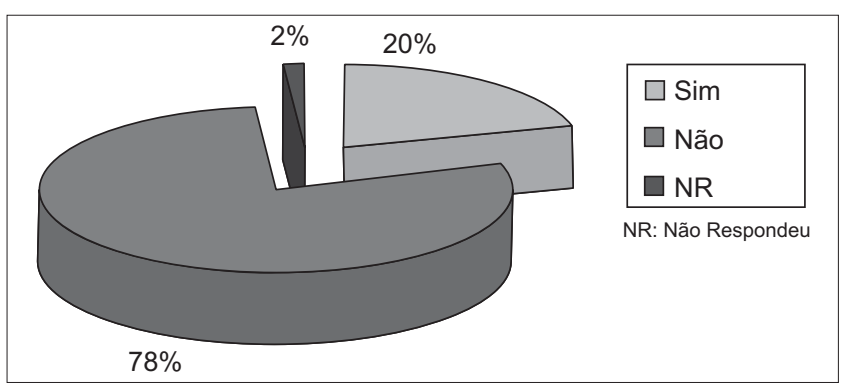

Gráfico 13. Prevalência de ingestão de álcool.

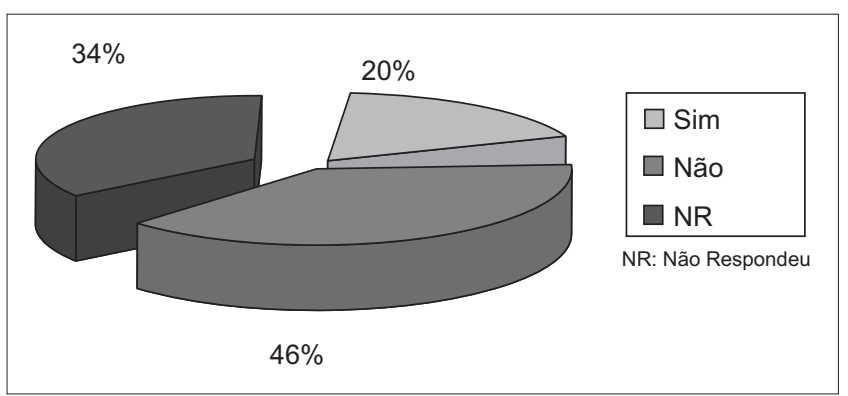

Gráfico 14. Prevalência do uso de medicamentos.

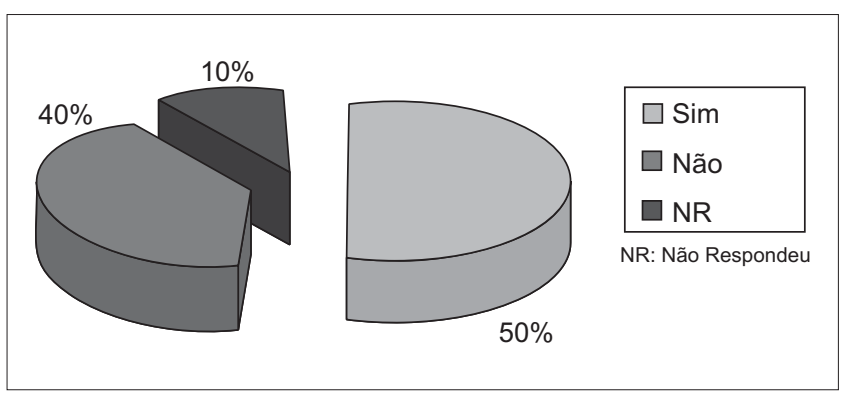

Gráfico 15. Prevalência da prática de atividade esportiva.
Assim fica evidente a diversidade da prevalência de cada um dos sintomas que compõem a SDO o que justifica os diferentes valores de ocorrência de disfonia em professores apresentada na literatura.

A auto-avaliação apresenta características próprias dirigidas às necessidades locais assim como visa fornecer elementos de controle epidemiológico.

A introdução de novas perguntas ao texto sempre foi precedida de discussão com a Comissão Tripartite e resultaram das dificuldades e observações que tivemos durante o trabalho prático e sua aplicação, sempre visando manter a objetividade e a simplicidade de entendimento com o mínimo dispêndio de tempo para a sua aplicação.

Por tratar-se de uma ação básica de saúde, deve ser utilizada pelo médico do trabalho que mantém o controle epidemiológico da população e para o otorrinolaringologista é um elemento inicial não restritivo à indicação dos procedimentos mais complexos.

Através dos gráficos podemos definir características da população propondo ações de saúde e para os indivíduos testados, podemos acrescentar ao controle clínico os dados obtidos e segui-los prospectivamente.

Em função destes achados podemos afirmar que a partir de um quadro de auto-avaliação devemos fazer propostas que atuem no sentido de reduzir a prevalência da SDO prevenindo assim o surgimento das laringopatias incapacitantes.

A elaboração da auto-avaliação com consenso técnico e social mostrou-se adequada pois além de ser um procedimento não oneroso é também não invasivo e abole técnicas que possam tornar-se discriminatórias.

\section{CONCLUSÃO}

O questionário de auto-avaliação para professores proposto pela Comissão Tripartite para Normatização da Voz Profissional mostrou-se satisfatório ao fornecer as prevalências de parâmetros que permitem a análise epidemiológica da síndrome disfônica ocupacional em professor.

\section{REFERÊNCIAS BIBLIOGRÁFICAS}

1. Leavell H \& Clark EG. Medicina Preventiva. McGrawHill do Brasil, São Paulo, 1976.

2. Dejonckere P H et al. A basic protocol for functional 
assesment of voice pathology especially for investigating the efficacy of (phonosurgicals) treatments and evaluating new asesment techniques. Eur Arch Otorhinolaryngol. 2001, 258:77-82.

3. Smith E et al. Frequency and effects of teachers voice problems. J Voice. 1997, 2:81-87.

4. Russel A, Oates J, Greenwood K. Prevalence of voice problems in teachers. J Voice. 1998, 12:467-479.
5. Ministério do Trabalho e Emprego (2002) Comissão Tripartite de Normatização para a Voz Profissional. Questionário de auto-avaliação para professores. Imprensa Oficial do Estado. 11 páginas.

6. Ministério do Trabalho e Emprego. Portaria 3214.

7. Calas M et al. La pathologie vocale chez l'enseignant. Rev Laryngol. 1989, 110:397-406. 